International Journal of Pure and Applied Mathematics

Volume 108 No. 1 2016, 169-183

ISSN: 1311-8080 (printed version); ISSN: 1314-3395 (on-line version)

url: http://www.ijpam.eu

doi: $10.12732 /$ ijpam.v108i1.15

\title{
INTEGRAL OSCILLATION CRITERIA FOR THIRD-ORDER DIFFERENTIAL EQUATIONS WITH DELAY ARGUMENT
}

\author{
Jozef Džurina ${ }^{1}$, Blanka Baculíkováa ${ }^{2}$, Irena Jadlovská $\$ 3$ \\ $1,2,3$ Department of Mathematics and Theoretical Informatics \\ Faculty of Electrical Engineering and Informatics \\ Technical University of Košice \\ Letná 9, 04200 Košice, SLOVAKIA
}

Abstract: In the present paper, some new criteria for property $\mathrm{A}$ and the oscillation of third order nonlinear delay differential equations of the type

$$
\left(a(t)\left[\left(b(t) y^{\prime}(t)\right)^{\prime}\right]^{\vee}\right)^{\prime}+p(t) f(y(\tau(t)))=0 .
$$

are established.

AMS Subject Classification: 34C10, 34K11

Key Words: third-order functional differential equations, property A, oscillation, delay argument, integral criteria

\section{Introduction}

We consider the nonlinear third-order delay differential equation

$$
\left(a(t)\left[\left(b(t) y^{\prime}(t)\right)^{\prime}\right]^{\gamma}\right)^{\prime}+p(t) f(y(\tau(t)))=0, t \geq t_{0} .
$$

In the sequel, it is always assumed that

$\left(H_{0}\right) \gamma$ is the ratio of odd positive integers,

$\left(H_{1}\right) \quad a, b, p \in C\left(\left[t_{0}, \infty\right), \mathbb{R}^{+}\right), R^{+}=(0, \infty)$,

Received: February 10, 2016

Published: June 6, 2016

${ }^{\S}$ Correspondence author (c) 2016 Academic Publications, Ltd.

url: www.acadpubl.eu 
$\left(H_{2}\right) \tau(t) \in C\left(\left[t_{0}, \infty\right), \mathbb{R}^{+}\right), \tau(t) \leq t, \tau(t)$ is nondecreasing and $\lim _{t \rightarrow \infty} \tau(t)=\infty$.

$\left(H_{3}\right) f(u) \in C(\mathbb{R}), u f(u)>0$ for $u \neq 0, f(u v) \geq f(u) f(v)$ for $u v>0, f$ is nondecreasing.

We further assume that $(E)$ is in canonical form, that is,

$$
\int_{t_{0}}^{\infty} a^{-1 / \gamma}(t) \mathrm{d} t=\infty, \quad \int_{t_{0}}^{\infty} b^{-1}(t) \mathrm{d} t=\infty
$$

By a solution of equation $(E)$, we mean a function $y(t) \in C^{1}\left(\left[T_{y}, \infty\right)\right), \quad T_{y} \geq t_{0}$, which has the property $a(t)\left[\left(b(t) y^{\prime}(t)\right)^{\prime}\right]^{\gamma} \in C^{1}\left(\left[T_{y}, \infty\right)\right)$ satisfies the equation $(E)$ on $\left[T_{y}, \infty\right)$. We consider only those solutions $y(t)$ of $(E)$ which satisfy

$$
\sup \{|y(t)|: t \geq T\}>0
$$

for all $T \geq T_{y}$. We assume that $(E)$ possesses such a solution. A solution of $(E)$ is called oscillatory if it has arbitrarily large zeros on $\left[T_{y}, \infty\right)$ and otherwise, it is called to be nonoscillatory. An equation is said to be oscillatory if all its solutions are oscillatory.

Differential equations of third order have long been considered as valuable tools in the modeling of many phenomena in different areas of applied mathematics and physics. For instance, such equations are encountered in the study of entry-flow phenomenon [4], the propagation of electrical pulses in the nerve of a squid approximated by the famous Nagumo's equation [10], the feedback nuclear reactor problem [12], the regulation of a steam turbine [8] and so on.

Hence, a great deal of work has been done in recent decades and the investigation of oscillatory and asymptotic properties for these equations has taken the shape of a well-developed theory turned mainly toward functional differential equations. In fact, the development of oscillation theory for the third order differential equations began in 1961 with the appearance of the work of Hanan [3] and Lazer [7]. Since then, many authors contributed to the subject studying different classes of equations and applying various techniques. A systematic survey of the most significant efforts in this theory can be found in the excellent monographs of Swanson [11], Greguš [2] and the very recent-one of Padhi and Pati [9].

Motivated by recent oscillation results of Koplatadze [6] exploited for higherorder differential equations with deviating argument of the type

$$
y^{(n)}(t)+q(t) y(\tau(t))=0,
$$


we derive in the paper some useful monotonic properties of nonoscillatory solutions which permit us to achieve such new sufficient conditions for $(E)$ to have property A or to be oscillatory that are different from most known.

As is convenient, we state here that all functional inequalities considered in this article are assumed to hold eventually, i.e., they are satisfied for all $t$ large enough.

\section{Preliminary Results}

We start with the classification of possible nonoscillatory solutions of $(E)$. Without loss of generality we can deal only with eventually positive solutions of $(E)$.

Lemma 1. Assume that $y(t)$ is an eventually positive solution of $(E)$. Then $y(t)$ satisfies one of the following conditions

$$
\begin{aligned}
& y^{\prime}(t)<0,\left(b(t) y^{\prime}(t)\right)^{\prime}>0,\left(a(t)\left[\left(b(t) y^{\prime}(t)\right)^{\prime}\right]^{\gamma}\right)^{\prime}<0, \\
& y^{\prime}(t)>0,\left(b(t) y^{\prime}(t)\right)^{\prime}>0,\left(a(t)\left[\left(b(t) y^{\prime}(t)\right)^{\prime}\right]^{\gamma}\right)^{\prime}<0,
\end{aligned}
$$

eventually.

Proof. The proof follows immediately from the canonical form of $(E)$ and so we omit it.

The following result presents very useful monotonic properties of nonoscillatory solutions of $(E)$. For a sake of brevity, we define the functions

$$
\begin{gathered}
A(t)=\int_{t_{*}}^{t} \frac{1}{a^{1 / \gamma}(s)} \mathrm{d} s, \\
B(t)=\int_{t_{*}}^{t} \frac{1}{b(s)} \mathrm{d} s, \\
C(t)=\int_{t_{*}}^{t} \frac{1}{b(u)} \int_{t_{*}}^{u} \frac{1}{a^{1 / \gamma}(s)} \mathrm{d} s \mathrm{~d} u,
\end{gathered}
$$

where $t_{*}$ is large enough. 
Lemma 2. Let $y(t)$ be a positive solution of $(E)$ satisfying $\left(\mathcal{N}_{2}\right)$ and

$$
\int_{t_{*}}^{\infty} \frac{1}{a^{1 / \gamma}(u)}\left[\int_{u}^{\infty} p(s) f(B(\tau(s))) \mathrm{d} s\right]^{1 / \gamma}=\infty
$$

Then

(i) $\frac{y(t)}{B(t)}$ is increasing,

(ii) $\frac{y(t)}{C(t)}$ is decreasing,

(iii) $\frac{b(t) y^{\prime}(t)}{A(t)}$ is decreasing.

Proof. Assume on the contrary that $(E)$ possesses an eventually positive solution $y(t)$ satisfying $\left(\mathcal{N}_{2}\right)$ for $t \geq t_{*}$. It follows from Lemma 1 that $a^{1 / \gamma}(t)\left(b(t) y^{\prime}(t)\right)^{\prime}$ is decreasing, thus,

$$
\begin{aligned}
b(t) y^{\prime}(t) & \geq \int_{t_{*}}^{t} a^{1 / \gamma}(s)\left(b(s) y^{\prime}(s)\right)^{\prime} \frac{1}{a^{1 / \gamma}(s)} \mathrm{d} s \\
& \geq a^{1 / \gamma}(t)\left(b(t) y^{\prime}(t)\right)^{\prime} \int_{t_{*}}^{t} \frac{1}{a^{1 / \gamma}(s)} \mathrm{d} s .
\end{aligned}
$$

This yields

$$
\left(\frac{b(t) y^{\prime}(t)}{A(t)}\right)^{\prime}=\frac{\left(b(t) y^{\prime}(t)\right)^{\prime} A(t)-b(t) y^{\prime}(t) \frac{1}{a^{1 / \mathrm{\gamma}}(t)}}{A^{2}(t)} \leq 0 .
$$

Consequently, $\frac{b(t) y^{\prime}(t)}{A(t)}$ is decreasing and, what is more,

$$
\begin{aligned}
y(t) & \geq \int_{t_{*}}^{t} \frac{b(u) y^{\prime}(u)}{A(u)} \frac{A(u)}{b(u)} \mathrm{d} u \\
& \geq \frac{b(t) y^{\prime}(t)}{A(t)} \int_{t_{*}}^{t} \frac{1}{b(u)} \int_{t_{*}}^{u} \frac{1}{a^{1 / \gamma}(s)} \mathrm{d} s \mathrm{~d} u .
\end{aligned}
$$

So, we deduce

$$
\left(\frac{y(t)}{C(t)}\right)^{\prime}=\frac{y^{\prime}(t) C(t)-y(t) A(t) \frac{1}{b(t)}}{C^{2}(t)} \leq 0
$$


which implies that $\frac{y(t)}{C(t)}$ is decreasing.

On the other hand, since $b(t) y^{\prime}(t)$ is increasing for any $t \geq t_{*}$, it is easy to see that

$$
\begin{aligned}
y(t) & =y\left(t_{1}\right)+\int_{t_{1}}^{t} \frac{b(s) y^{\prime}(s)}{b(s)} \mathrm{d} s \leq y\left(t_{1}\right)+b(t) y^{\prime}(t) \int_{t_{1}}^{t} \frac{1}{b(s)} \mathrm{d} s \\
& =y\left(t_{1}\right)-b(t) y^{\prime}(t) \int_{t_{*}}^{t_{1}} \frac{1}{b(s)} \mathrm{d} s+b(t) y^{\prime}(t) \int_{t_{*}}^{t} \frac{1}{b(s)} \mathrm{d} s,
\end{aligned}
$$

for all $t \geq t_{1}>t_{*}$. Condition (2) implies that $b(t) y^{\prime}(t) \rightarrow \infty$ as $t \rightarrow \infty$. Therefore, there exists a $t_{2}>t_{1}$ such that for any $t \geq t_{2}$

$$
y(t) \leq b(t) y^{\prime}(t) \int_{t_{*}}^{t} \frac{1}{b(s)} \mathrm{d} s .
$$

Using this fact, we arrive at

$$
\left(\frac{y(t)}{B(t)}\right)^{\prime}=\frac{y^{\prime}(t) B(t)-y(t) \frac{1}{b(t)}}{B^{2}(t)} \geq 0
$$

eventually and we conclude that $\frac{y(t)}{B(t)}$ is increasing. The proof is complete.

The following result is elementary but useful in what comes next.

Lemma 3. Assume $A \geq 0, B \geq 0, \alpha \geq 1$. Then

$$
(A+B)^{\alpha} \geq A^{\alpha}+B^{\alpha} \text {. }
$$

Proof. If $A=0$ or $B=0$, then (5) holds. For $A \neq 0$, we set $x=B / A$ and the condition (5) takes the form $(1+x)^{\alpha} \geq 1+x^{\alpha}$, which is for $x>0$ evidently true.

Lemma 4. Assume $A \geq 0, B \geq 0,0<\alpha \leq 1$. Then

$$
(A+B)^{\alpha} \geq \frac{A^{\alpha}+B^{\alpha}}{2^{1-\alpha}} \text {. }
$$

Proof. We may assume that $0<A<B$. Consider a function $g(u)=u^{\alpha}$. Since $g^{\prime \prime}(u)<0$ for $u>0$, the function $g(u)$ is concave down, that is,

$$
g\left(\frac{A+B}{2}\right) \geq \frac{g(A)+g(B)}{2},
$$


which implies (6).

To simplify our formulations of the main results, we recall the following definition.

Definition 1. We say that $(E)$ enjoys property A if every its nonoscillatory solution satisfies $\left(\mathcal{N}_{0}\right)$.

Property A of third order differential equations has been widely studied in the literature, see $[1,5]$ and references cited therein.

\section{Criteria for Property A of $(E)$}

Employing our lemmas, we provide in this section several limsup type criteria for $(E)$ to have property A.

Theorem 2. Let (2) hold, $\gamma \in(0,1\rangle$ and

$$
\lim _{u \rightarrow \pm \infty} \frac{u}{f^{1 / \gamma}(u)}=K_{1}<\infty .
$$

If

$$
\begin{aligned}
\limsup _{t \rightarrow \infty}\{ & \frac{C(\tau(t))}{A(\tau(t))} f^{1 / \gamma}\left(\frac{1}{C(\tau(t))}\right) \int_{t_{*}}^{\tau(t)} \frac{1}{a^{1 / \gamma}(u)} \times \\
\times & {\left[\int_{u}^{\tau(t)} p(s) f(C(\tau(s))) \mathrm{d} s\right]^{1 / \gamma} \mathrm{d} u } \\
& +C(\tau(t)) f^{1 / \gamma}\left(\frac{1}{C(\tau(t))}\right)\left[\int_{\tau(t)}^{t} p(s) f(C(\tau(s))) \mathrm{d} s\right]^{1 / \gamma} \\
& \left.+C(\tau(t)) f^{1 / \gamma}\left(\frac{1}{B(\tau(t))}\right)\left[\int_{t}^{\infty} p(s) f(B(\tau(s))) \mathrm{d} s\right]^{1 / \gamma}\right\}>K_{1},
\end{aligned}
$$

then $(E)$ has property $A$.

Proof. Assume on the contrary that $(E)$ possesses an eventually positive solution $y(t)$ satisfying $\left(\mathcal{N}_{2}\right), t \geq t_{*}$. An integration of $(E)$ from $t$ to $\infty$ yields

$$
\left[\left(b(t) y^{\prime}(t)\right)^{\prime}\right]^{\gamma} \geq \frac{1}{a(t)} \int_{t}^{\infty} p(s) f(y(\tau(s))) \mathrm{d} s .
$$


Integrating from $t_{*}$ to $t$, one gets

$$
\begin{gathered}
b(t) y^{\prime}(t) \geq \int_{t_{*}}^{t} \frac{1}{a^{1 / \gamma}(u)}\left[\int_{u}^{\infty} p(s) f(y(\tau(s))) \mathrm{d} s\right]^{1 / \gamma} \mathrm{d} u \\
=\int_{t_{*}}^{t} \frac{1}{a^{1 / \gamma}(u)}\left[\int_{u}^{t} p(s) f(y(\tau(s))) \mathrm{d} s+\int_{t}^{\infty} p(s) f(y(\tau(s))) \mathrm{d} s\right]^{1 / \gamma} \mathrm{d} u .
\end{gathered}
$$

Since $\gamma \in(0,1\rangle$, Lemma 3 implies

$$
\begin{aligned}
b(t) y^{\prime}(t) \geq & \int_{t_{*}}^{t} \frac{1}{a^{1 / \gamma}(u)}\left[\int_{u}^{t} p(s) f(y(\tau(s))) \mathrm{d} s\right]^{1 / \gamma} \mathrm{d} u \\
& +\int_{t_{*}}^{t} \frac{1}{a^{1 / \gamma}(u)}\left[\int_{t}^{\infty} p(s) f(y(\tau(s))) \mathrm{d} s\right]^{1 / \gamma} \mathrm{d} u \\
& =\int_{t_{*}}^{t} \frac{1}{a^{1 / \gamma}(u)}\left[\int_{u}^{t} p(s) f(y(\tau(s))) \mathrm{d} s\right]^{1 / \gamma} \mathrm{d} u \\
& +A(t)\left[\int_{t}^{\infty} p(s) f(y(\tau(s))) \mathrm{d} s\right]^{1 / \gamma} .
\end{aligned}
$$

Taking (4) into account, one can see

$$
\begin{aligned}
\frac{A(t) y(t)}{C(t)} & \geq \int_{t_{*}}^{t} \frac{1}{a^{1 / \gamma}(u)}\left[\int_{u}^{t} p(s) f(y(\tau(s))) \mathrm{d} s\right]^{1 / \gamma} \mathrm{d} u \\
& +A(t)\left[\int_{t}^{\infty} p(s) f(y(\tau(s))) \mathrm{d} s\right]^{1 / \gamma}
\end{aligned}
$$

or

$$
\begin{aligned}
\frac{A(\tau(t)) y(\tau(t))}{C(\tau(t))} \geq & \int_{t_{*}}^{\tau(t)} \frac{1}{a^{1 / \gamma}(u)}\left[\int_{u}^{\tau(t)} p(s) f(y(\tau(s))) \mathrm{d} s\right]^{1 / \gamma} \mathrm{d} u \\
& +A(\tau(t))\left[\int_{\tau(t)}^{t} p(s) f(y(\tau(s))) \mathrm{d} s\right]^{1 / \gamma} \\
& +A(\tau(t))\left[\int_{t}^{\infty} p(s) f(y(\tau(s))) \mathrm{d} s\right]^{1 / \gamma}
\end{aligned}
$$

Taking the monotonicity properties $(i)$ - $($ iii $)$ of Lemma 2 and $\left(H_{3}\right)$ into account, 
one can verify that

$$
\begin{gathered}
\frac{A(\tau(t)) y(\tau(t))}{C(\tau(t))} \geq \\
f^{1 / \gamma}\left(\frac{y(\tau(t))}{C(\tau(t))}\right) \int_{t_{*}}^{\tau(t)} \frac{1}{a^{1 / \gamma}(u)}\left[\int_{u}^{\tau(t)} p(s) f(C(\tau(s))) \mathrm{d} s\right]^{1 / \gamma} \mathrm{d} u \\
+A(\tau(t)) f^{1 / \gamma}\left(\frac{y(\tau(t))}{C(\tau(t))}\right)\left[\int_{\tau(t)}^{t} p(s) f(C(\tau(s))) \mathrm{d} s\right]^{1 / \gamma} \\
+A(\tau(t)) f^{1 / \gamma}\left(\frac{y(\tau(t))}{B(\tau(t))}\right)\left[\int_{t}^{\infty} p(s) f(B(\tau(s))) \mathrm{d} s\right]^{1 / \gamma}
\end{gathered}
$$

which in view of $\left(H_{3}\right)$ yields

$$
\begin{gathered}
\frac{y(\tau(t))}{f^{1 / \gamma}(y(\tau(t)))} \geq \\
\frac{C(\tau(t))}{A(\tau(t))} f^{1 / \gamma}\left(\frac{1}{C(\tau(t))}\right) \int_{t_{*}}^{\tau(t)} \frac{1}{a^{1 / \gamma}(u)}\left[\int_{u}^{\tau(t)} p(s) f(C(\tau(s))) \mathrm{d} s\right]^{1 / \gamma} \mathrm{d} u \\
+C(\tau(t)) f^{1 / \gamma}\left(\frac{1}{C(\tau(t))}\right)\left[\int_{\tau(t)}^{t} p(s) f(C(\tau(s))) \mathrm{d} s\right]^{1 / \gamma} \\
+C(\tau(t)) f^{1 / \gamma}\left(\frac{1}{B(\tau(t))}\right)\left[\int_{t}^{\infty} p(s) f(B(\tau(s))) \mathrm{d} s\right]^{1 / \gamma}
\end{gathered}
$$

Taking limsup as $t \rightarrow \infty$ on both sides of the previous inequality, we are led to the contradiction with assumptions of the theorem. The proof is complete.

The criterion obtained covers super-linear and half-linear case of $(E)$. In the following corollaries, it is always assumed that $\delta$ is the ratio of odd positive integers. 
Corollary 3. Let (2) hold, $\gamma \in(0,1)$ and

$$
\begin{aligned}
\limsup _{t \rightarrow \infty}\{ & \frac{C^{1-\frac{\delta}{\mathrm{V}}}(\tau(t))}{A(\tau(t))} \int_{t_{*}}^{\tau(t)} \frac{1}{a^{1 / \gamma}(u)}\left[\int_{u}^{\tau(t)} p(s) C^{\delta}(\tau(s)) \mathrm{d} s\right]^{1 / \gamma} \mathrm{d} u \\
& +C^{1-\frac{\delta}{\mathrm{V}}}(\tau(t))\left[\int_{\tau(t)}^{t} p(s) C^{\delta}(\tau(s)) \mathrm{d} s\right]^{1 / \gamma} \\
& \left.+\frac{C(\tau(t))}{B^{\frac{\delta}{\mathrm{V}}}(\tau(t))}\left[\int_{t}^{\infty} p(s) B^{\delta}(\tau(s)) \mathrm{d} s\right]^{1 / \gamma}\right\}>0,
\end{aligned}
$$

then the differential equation

$$
\left[a(t)\left(b(t)\left(y^{\prime}(t)\right)^{\gamma}\right)^{\prime}\right]^{\prime}+p(t) y^{\delta}(\tau(t))=0, \quad \delta>\gamma,
$$

has property $A$.

Corollary 4. Let (2) hold, $\gamma \in(0,1)$ and

$$
\begin{gathered}
\limsup _{t \rightarrow \infty}\left\{\frac{1}{A(\tau(t))} \int_{t_{*}}^{\tau(t)} \frac{1}{a^{1 / \gamma}(u)}\left[\int_{u}^{\tau(t)} p(s) C^{\gamma}(\tau(s)) \mathrm{d} s\right]^{1 / \gamma} \mathrm{d} u\right. \\
+\left[\int_{\tau(t)}^{t} p(s) C^{\gamma}(\tau(s)) \mathrm{d} s\right]^{1 / \gamma} \\
\left.+\frac{C(\tau(t))}{B(\tau(t))}\left[\int_{t}^{\infty} p(s) B^{\gamma}(\tau(s)) \mathrm{d} s\right]^{1 / \gamma}\right\}>1
\end{gathered}
$$

then the differential equation

$$
\left[a(t)\left(b(t)\left(y^{\prime}(t)\right)^{\gamma}\right)^{\prime}\right]^{\prime}+p(t) y^{\gamma}(\tau(t))=0
$$

has property $A$.

We are about to provide another criterion for property A that is applicable when $(E)$ is of sub-linear type.

Theorem 5. Let (2) hold, $\gamma \in(0,1)$ and

$$
\int_{t_{1}}^{\infty} p(s) f(C(\tau(s))) \mathrm{d} s=\infty .
$$


Assume that

$$
\lim _{u \rightarrow 0} \frac{u}{f^{1 / \gamma}(u)}=K_{2}<\infty
$$

If

$$
\begin{aligned}
\limsup _{t \rightarrow \infty}\left\{\frac{1}{A(\tau(t))} \int_{t_{*}}^{\tau(t)} \frac{1}{a^{1 / \gamma}(u)}\left[\int_{u}^{\tau(t)} p(s) f(C(\tau(s))) \mathrm{d} s\right]^{1 / \gamma} \mathrm{d} u\right. \\
+\left[\int_{\tau(t)}^{t} p(s) f(C(\tau(s))) \mathrm{d} s\right]^{1 / \gamma} \\
\left.+f^{1 / \gamma}\left(\frac{C(\tau(t))}{B(\tau(t))}\right)\left[\int_{t}^{\infty} p(s) f(B(\tau(s))) \mathrm{d} s\right]^{1 / \gamma}\right\}>K_{2}
\end{aligned}
$$

then $(E)$ has property $A$.

Proof. Assume that $(E)$ has an eventually positive solution $y(t)$ satisfying $\left(\mathcal{N}_{2}\right)$ for any $t \geq t_{*}$. We claim that $(9)$ implies

$$
\lim _{t \rightarrow \infty} \frac{y(t)}{C(t)}=0 \text {. }
$$

Assume the the contrary, that is $\lim _{t \rightarrow \infty} \frac{y(t)}{C(t)}=\ell>0$. By the L'Hospital rule

$$
\ell=\lim _{t \rightarrow \infty} \frac{y(t)}{C(t)}=\lim _{t \rightarrow \infty} a^{1 / \gamma}(t)\left[b(t) y^{\prime}(t)\right]^{\prime} .
$$

Combining (3), (4) and (11), one gets

$$
y(t) \geq C(t)\left\{a^{1 / \gamma}(t)\left[b(t) y^{\prime}(t)\right]^{\prime}\right\} \geq \ell C(t) .
$$

On the other hand, an integration of $(E)$ from $t_{*}$ to $\infty$ yields

$$
k=\left.a(s)\left[\left(b(s) y^{\prime}(s)\right)^{\prime}\right]^{\gamma}\right|_{s=t_{*}} \geq \int_{t_{1}}^{\infty} p(s) f(y(\tau(s))) \mathrm{d} s,
$$

which in view of (12) gives

$$
k \geq f(\ell) \int_{t_{1}}^{\infty} p(s) f(C(\tau(s))) \mathrm{d} s .
$$

This contradicts with (9) and we conclude that $y(t) / C(t) \rightarrow 0$ as $t \rightarrow \infty$. 
Now, setting

$$
w(t)=\frac{y(\tau(t))}{C(\tau(t))},
$$

the condition (8) together with $\left(H_{3}\right)$ implies

$$
\begin{aligned}
\frac{w(t)}{f^{1 / \gamma}(w(t))} & \geq \\
& \frac{1}{A(\tau(t))} \int_{t_{*}}^{\tau(t)} \frac{1}{a^{1 / \gamma}(u)}\left[\int_{u}^{\tau(t)} p(s) f(C(\tau(s))) \mathrm{d} s\right]^{1 / \gamma} \mathrm{d} u \\
+ & {\left[\int_{\tau(t)}^{t} p(s) f(C(\tau(s))) \mathrm{d} s\right]^{1 / \gamma} } \\
& +f^{1 / \gamma}\left(\frac{C(\tau(t))}{B(\tau(t))}\right)\left[\int_{t}^{\infty} p(s) f(B(\tau(s))) \mathrm{d} s\right]^{1 / \gamma} .
\end{aligned}
$$

Taking limsup as $t \rightarrow \infty$ on both sides of the previous inequality, we are led to contradiction with assumptions of our theorem. The proof is complete.

For the half-linear case $f(u)=u^{\gamma}$, Theorem 2 reduces to Corollary 2, while in sub-linear case, we have the following result.

Corollary 6. Let (2) and (9) hold and $\gamma \in(0,1\rangle$. If

$$
\begin{gathered}
\limsup _{t \rightarrow \infty}\left\{\frac{1}{A(\tau(t))} \int_{t_{*}}^{\tau(t)} \frac{1}{a^{1 / \gamma}(u)}\left[\int_{u}^{\tau(t)} p(s) C^{\delta}(\tau(s)) \mathrm{d} s\right]^{1 / \gamma} \mathrm{d} u\right. \\
+\left[\int_{\tau(t)}^{t} p(s) C^{\delta}(\tau(s)) \mathrm{d} s\right]^{1 / \gamma} \\
\left.+\left(\frac{C(\tau(t))}{B(\tau(t))}\right)^{\delta / \gamma}\left[\int_{t}^{\infty} p(s) B^{\delta}(\tau(s)) \mathrm{d} s\right]^{1 / \gamma}\right\}>0
\end{gathered}
$$

then the differential equation

$$
\left[a(t)\left(b(t)\left(y^{\prime}(t)\right)^{\gamma}\right)^{\prime}\right]^{\prime}+p(t) y^{\delta}(\tau(t))=0, \quad \gamma>\delta .
$$

has property $A$.

Considering Lemma 4 , all previous results can rewritten to cover the case when $\gamma \geq 1$. We provide the main results. 
Theorem 7. Let $\gamma \geq 1$ and $\alpha=2^{1 / \gamma-1}$. Assume that (2) and (7) hold. If

$$
\begin{gathered}
\limsup _{t \rightarrow \infty}\left\{\frac{\alpha C(\tau(t))}{A(\tau(t))} f^{1 / \gamma}\left(\frac{1}{C(\tau(t))}\right) \int_{t_{*}}^{\tau(t)} \frac{\left[\int_{u}^{\tau(t)} p(s) f(C(\tau(s))) \mathrm{d} s\right]^{1 / \gamma}}{a^{1 / \gamma}(u)} \mathrm{d} u\right. \\
+\alpha^{2} C(\tau(t)) f^{1 / \gamma}\left(\frac{1}{C(\tau(t))}\right)\left[\int_{\tau(t)}^{t} p(s) f(C(\tau(s))) \mathrm{d} s\right]^{1 / \gamma} \\
\left.+\alpha^{2} C(\tau(t)) f^{1 / \gamma}\left(\frac{1}{B(\tau(t))}\right)\left[\int_{t}^{\infty} p(s) f(B(\tau(s))) \mathrm{d} s\right]^{1 / \gamma}\right\}>K_{1},
\end{gathered}
$$

then (E) has property $A$.

Theorem 8. Let $\gamma \geq 1$ and $\alpha=2^{1 / \gamma-1}$. Assume that (2), (9), and (10) hold. If

$$
\begin{aligned}
\limsup _{t \rightarrow \infty} & \left\{\frac{\alpha}{A(\tau(t))} \int_{t_{*}}^{\tau(t)} \frac{1}{a^{1 / \gamma}(u)}\left[\int_{u}^{\tau(t)} p(s) f(C(\tau(s))) \mathrm{d} s\right]^{1 / \gamma} \mathrm{d} u\right. \\
& +\alpha^{2}\left[\int_{\tau(t)}^{t} p(s) f(C(\tau(s))) \mathrm{d} s\right]^{1 / \gamma} \\
& \left.+\alpha^{2} f^{1 / \gamma}\left(\frac{C(\tau(t))}{B(\tau(t))}\right)\left[\int_{t}^{\infty} p(s) f(B(\tau(s))) \mathrm{d} s\right]^{1 / \gamma}\right\}>K_{2},
\end{aligned}
$$

then $(E)$ has property $A$.

\section{Oscillation of $(E)$}

Due to the presence of the delay argument, we are also able to eliminate possible eventually positive solutions of $\left(\mathcal{N}_{0}\right)$-type and so attain oscillation of the equation $(E)$.

Theorem 9. Assume that

$$
\lim _{u \rightarrow 0} \frac{u}{f^{1 / \gamma}(u)}=K_{3}<\infty .
$$

If

$$
\limsup _{t \rightarrow \infty} \int_{\tau(t)}^{t} \frac{1}{b(v)} \int_{v}^{t} \frac{1}{a^{1 / \gamma}(u)}\left[\int_{u}^{t} p(s) \mathrm{d} s \mathrm{~d} u\right]^{1 / \gamma} \mathrm{d} v>K_{3},
$$


then $(E)$ does not possess any positive solution satisfying $\left(\mathcal{N}_{0}\right)$.

Proof. On the contrary, assume that $(E)$ possesses an eventually positive solution $y(t)$ satisfying $\left(\mathcal{N}_{0}\right)$, for any $t \geq t_{1}$. First, note that (14) implies

$$
\int_{t_{1}}^{\infty} \frac{1}{b(v)} \int_{v}^{\infty} \frac{1}{a^{1 / \gamma}(u)}\left[\int_{u}^{\infty} p(s) \mathrm{d} s \mathrm{~d} u\right]^{1 / \gamma} \mathrm{d} v=\infty
$$

which guarantees that $y(t) \rightarrow 0$ as $t \rightarrow \infty$.

On the other hand, integrating $(E)$ twice in $s$, from $s$ to $t>s$, one gets

$$
-y^{\prime}(s) \geq \frac{f^{1 / \gamma}(y(\tau(t)))}{b(s)} \int_{s}^{t} \frac{1}{a^{1 / \gamma}(u)}\left[\int_{u}^{t} p(x) \mathrm{d} x \mathrm{~d} u\right]^{1 / \gamma} .
$$

Integrating once more, we obtain

$$
y(s) \geq f^{1 / \gamma}(y(\tau(t))) \int_{s}^{t} \frac{1}{b(v)} \int_{v}^{t} \frac{1}{a^{1 / \gamma}(u)}\left[\int_{u}^{t} p(s) \mathrm{d} s \mathrm{~d} u\right]^{1 / \gamma} \mathrm{d} v .
$$

Setting $s=\tau(t)$, we get

$$
\frac{y(\tau(t))}{f^{1 / \gamma}(y(\tau(t)))} \geq \int_{\tau(t)}^{t} \frac{1}{b(v)} \int_{v}^{t} \frac{1}{a^{1 / \gamma}(u)}\left[\int_{u}^{t} p(s) \mathrm{d} s \mathrm{~d} u\right]^{1 / \gamma} \mathrm{d} v
$$

Taking limsup as $t \rightarrow \infty$ on both sides of the previous inequality, we are led to contradiction with (14). The proof is complete.

Combining results for nonexistence solutions of type $\left(\mathcal{N}_{2}\right)$ and $\left(\mathcal{N}_{0}\right)$ simultaneously, we immediately obtain criteria for oscillation of $(E)$.

Theorem 10. Let all conditions of Theorem 2 (Theorem 5, Theorem 7, Theorem 8) and Theorem 59 hold. Then $(E)$ is oscillatory.

We demonstrate our main results on the following illustrative example.

Example 1. We consider the third order delay differential equation

$$
\left[t^{1 / 4}\left(\left(t^{1 / 3} y^{\prime}(t)\right)^{\prime}\right)^{1 / 3}\right]^{\prime}+\frac{k}{t^{47 / 36}} y^{1 / 3}(\lambda t)=0, \quad t \geq 1
$$


where $k>0$ and $\lambda \in(0,1)$. Simple computation shows that

$$
A(t) \sim 4 t^{1 / 4}, \quad B(t) \sim \frac{3 t^{2 / 3}}{2}, \quad C(t) \sim \frac{48 t^{11 / 12}}{11} .
$$

It follows from Corollary 4 that condition

$$
k^{3} \frac{48}{11} \lambda^{11 / 12}\left(3264-\ln ^{3} \lambda\right)>1
$$

guarantees property $\mathrm{A}$ of $\left(E_{x}\right)$. For e.g. $\lambda=1 / 3$ it occurs if $k>0.057703$. And really, in the opposite cases if e.g. $k=0.0282$, then $\left(E_{x}\right)$ has not property $\mathrm{A}$, since it possesses a positive solution $y(t)=t^{0,766}$ satisfying $\left(\mathcal{N}_{2}\right)$.

Moreover, taking Theorem 9 into account, we are also able to eliminate positive solutions satisfying $\left(\mathcal{N}_{0}\right)$ provided that

$$
\begin{aligned}
k^{3}\left(\frac{36}{11}\right)^{3} & {\left[-\frac{3}{2} \ln \lambda-\frac{3888}{143}\left(1-\lambda^{11 / 36}\right)+\frac{972}{11}\left(1-\lambda^{11 / 18}\right)\right.} \\
& \left.+\frac{48}{11}\left(1-\lambda^{11 / 12}\right)-\frac{4317}{52}\left(1-\lambda^{2 / 3}\right)\right]>1,
\end{aligned}
$$

which guarantees oscillation of $\left(E_{x}\right)$. For e.g. $\lambda=1 / 3$ it occurs if $k>2.694$.

\section{Acknowledgements}

This work was supported by Slovak Research and Development Agency under contracts No. APVV-0404-12.

\section{References}

[1] M. Cecchi, Z. Došlá, M. Marini, On third order differential equations with Property A and B, Journal of Mathematical Analysis and Applications 231 (1999), 509-525.

[2] M. Greguš, Third order linear differential equations, Springer, 1987.

[3] M. Hanan, Oscillation criteria for third-order linear differential equations, Pacific Journal of Mathematics, 11(1961), 919-944.

[4] G. Jayaraman, N. Padmanabhan and R. Mehrotra, Entry flow into a circular tube of slowly varying cross section, Fluid Dynamics Research, 1(2) (1986), 131-144.

[5] I. T. Kiguradze, T. A. Chanturia, Asymptotic Properties of Solutions of Nonatunomous Ordinary Differential Equations, Kluwer Acad. Publ., Dordrecht 1993. 
[6] R. Koplatadze, G. Kvinkadze, I. P. Stavroulakis, Properties A and B of n-th order linear differential equations with deviating argument, Georgian Math. J. 6(1999), 553-566

[7] A. Lazer, The behavior of solutions of the differential equation, Pacific J. Math, 17(1966), pp. $430-435$.

[8] G. A. Munoz-Hernandez, D. Jones, Modelling and controlling hydropower plants. Springer Science \& Business Media, 2012. APA

[9] S. Padhi, S.Pati, Theory of third-order differential equations, Springer 2014.

[10] H. P. Mckean, Nagumo's equation, Pacific J. Math, 17(1966), pp. Advances in Mathematics 4, 209-223.

[11] Ch. A. Swanson, Comparison and oscillation theory of linear differential equations, Elsevier, 1968.

[12] S. D. Vreeke, G. M. Sandquist, Phase plane analysis of reactor kineticks, Neuclar Sci. Engner. 42 (1070), 259-305. 
